Supplement of The Cryosphere, 10, 341-358, 2016

http://www.the-cryosphere.net/10/341/2016/

doi:10.5194/tc-10-341-2016-supplement

(C) Author(s) 2016. CC Attribution 3.0 License.

(c) (i)

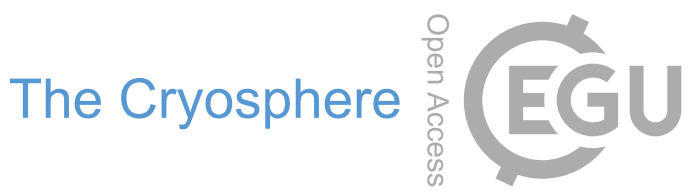

Supplement of

\title{
Effect of soil property uncertainties on permafrost thaw projections: a calibration-constrained analysis
}

\section{R. Harp et al.}

Correspondence to: D. R. Harp (dharp@lanl.gov)

The copyright of individual parts of the supplement might differ from the CC-BY 3.0 licence. 

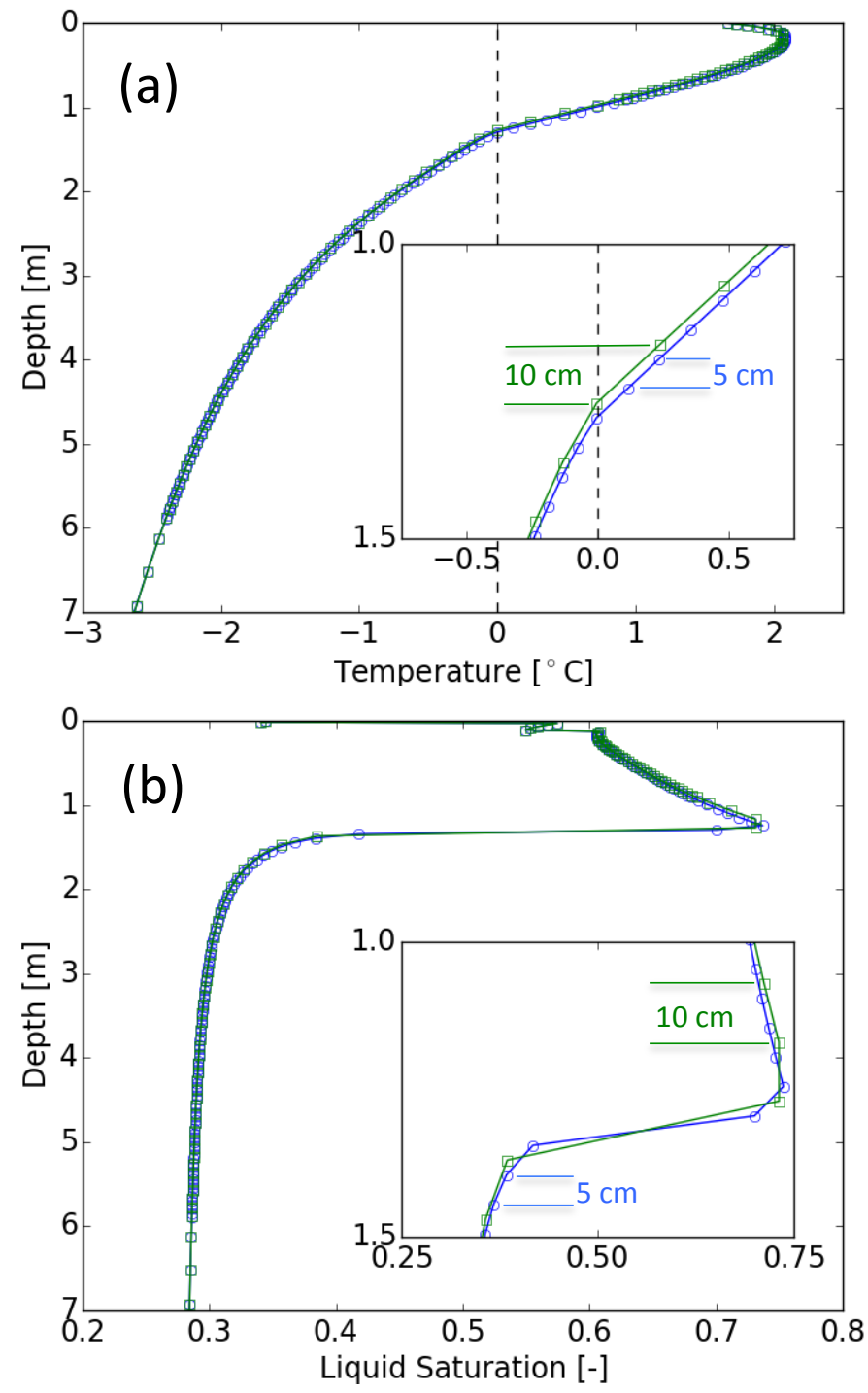

Figure S1: Evaluation of mesh discretization showing (a) temperature and (b) liquid saturation profiles. The profiles are from the ensemble member with the deepest simulated active layer in year 2100 ( $\sim 1.3 \mathrm{~m}$ deep), i.e., the ensemble member that will be most effected by mesh discretization. The ensemble member is run with a $10 \mathrm{~cm}$ (green boxes) and $5 \mathrm{~cm}$ cell (blue circles) spacing from $0.92 \mathrm{~m}$ to $5.92 \mathrm{~m}$. The insets allow visual inspection of the mesh spacings denoted by the symbols (boxs and circles). The small differences between the two cases for this worst case scenario indicate that the $10 \mathrm{~cm}$ spacing used in this research is adequate. 


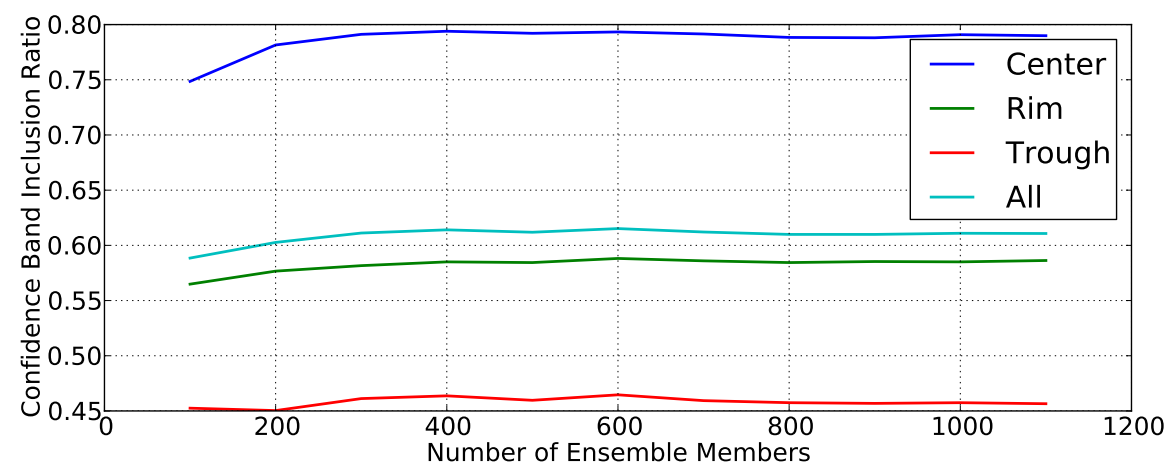

Figure S2: Calibration-constrained ensemble convergence analysis based on the ratio of measured temperatures from the $\mathrm{BEO}$ within the $95 \%$ confidence band for ensemble simulated temperatures. The relatively flat lines after around 800 ensemble members indicate convergence of the ensemble. 


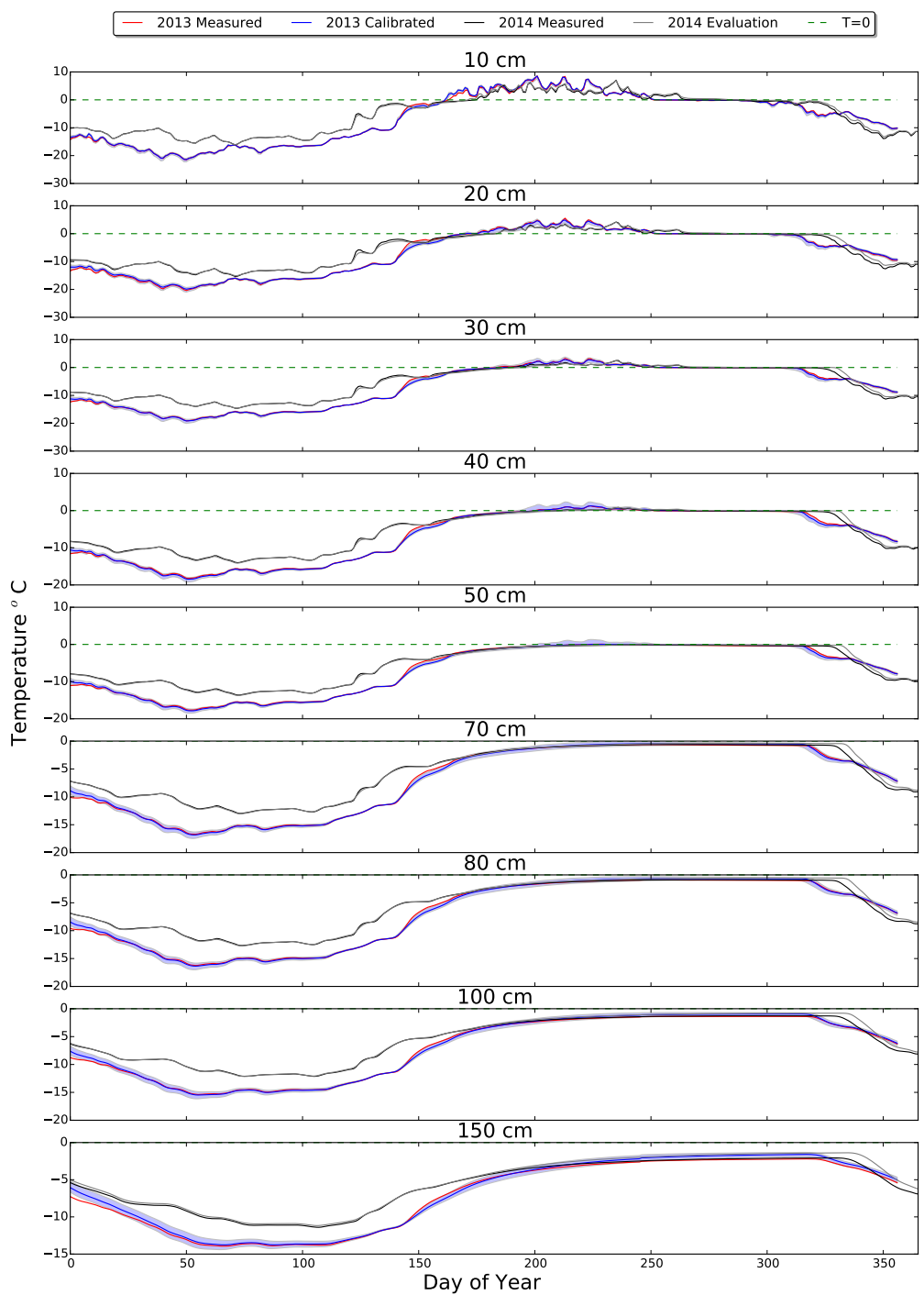

Figure S3: Time-series of temperature at specific depths for the polygonal center. Calendar year 2013 measured values from the BEO used as calibration targets are shown as a red line, the mean of the NSMC sample as a blue line, and the $95 \%$ confidence band is the shaded light blue region. Calendar year 2014 measured values from the BEO used as evaluation data for the 2013 calibration are shown as a black line. The gray line represents the 2014 simulated temperatures. 


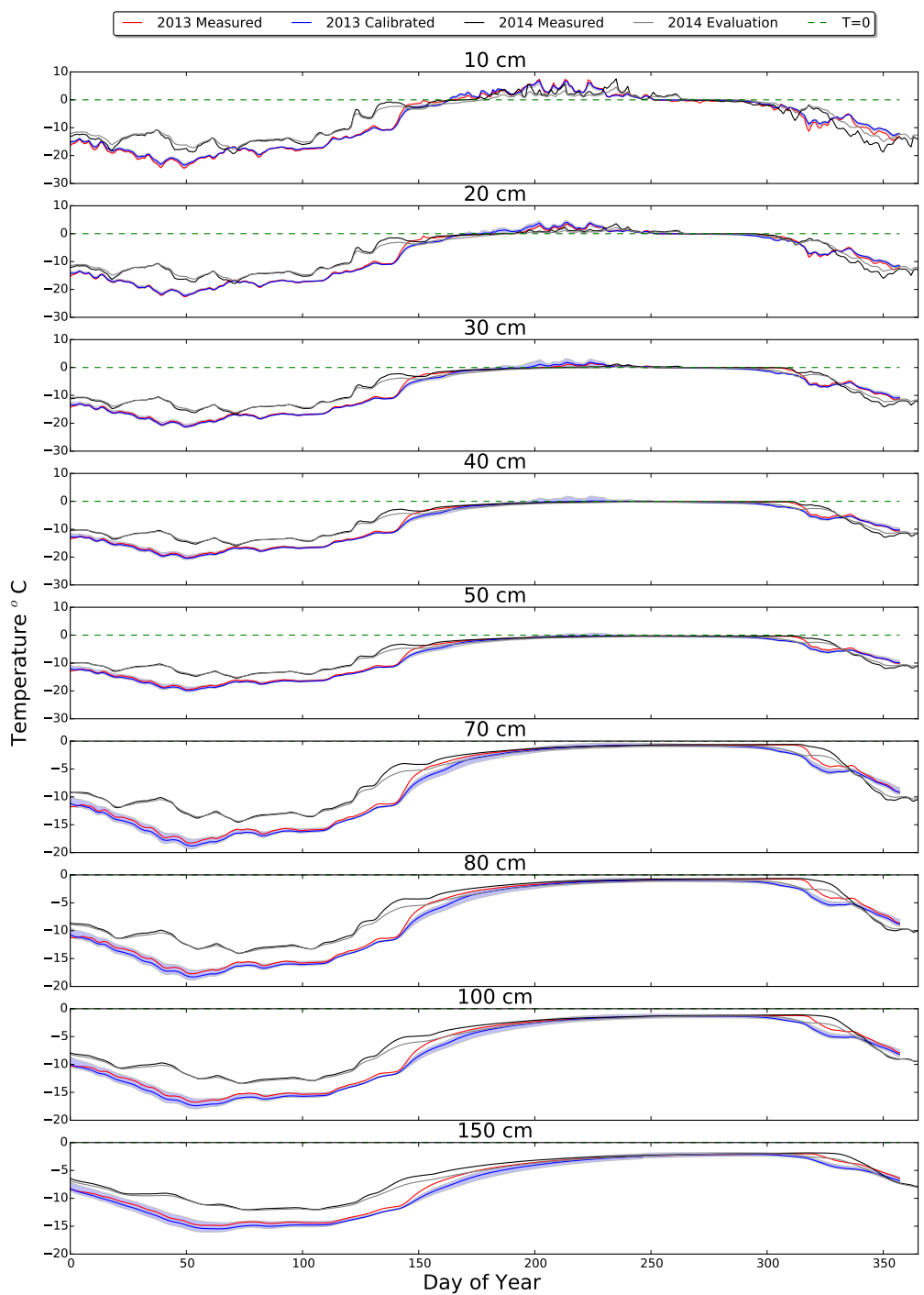

Figure S4: Time-series of temperature at specific depths for the polygonal rim. Calendar year 2013 measured values from the BEO used as calibration targets are shown as a red line, the mean of the NSMC sample as a blue line, and the $95 \%$ confidence band is the shaded light blue region. Calendar year 2014 measured values from the $\mathrm{BEO}$ used as evaluation data for the 2013 calibration are shown as a black line. The gray line represents the 2014 simulated temperatures. 


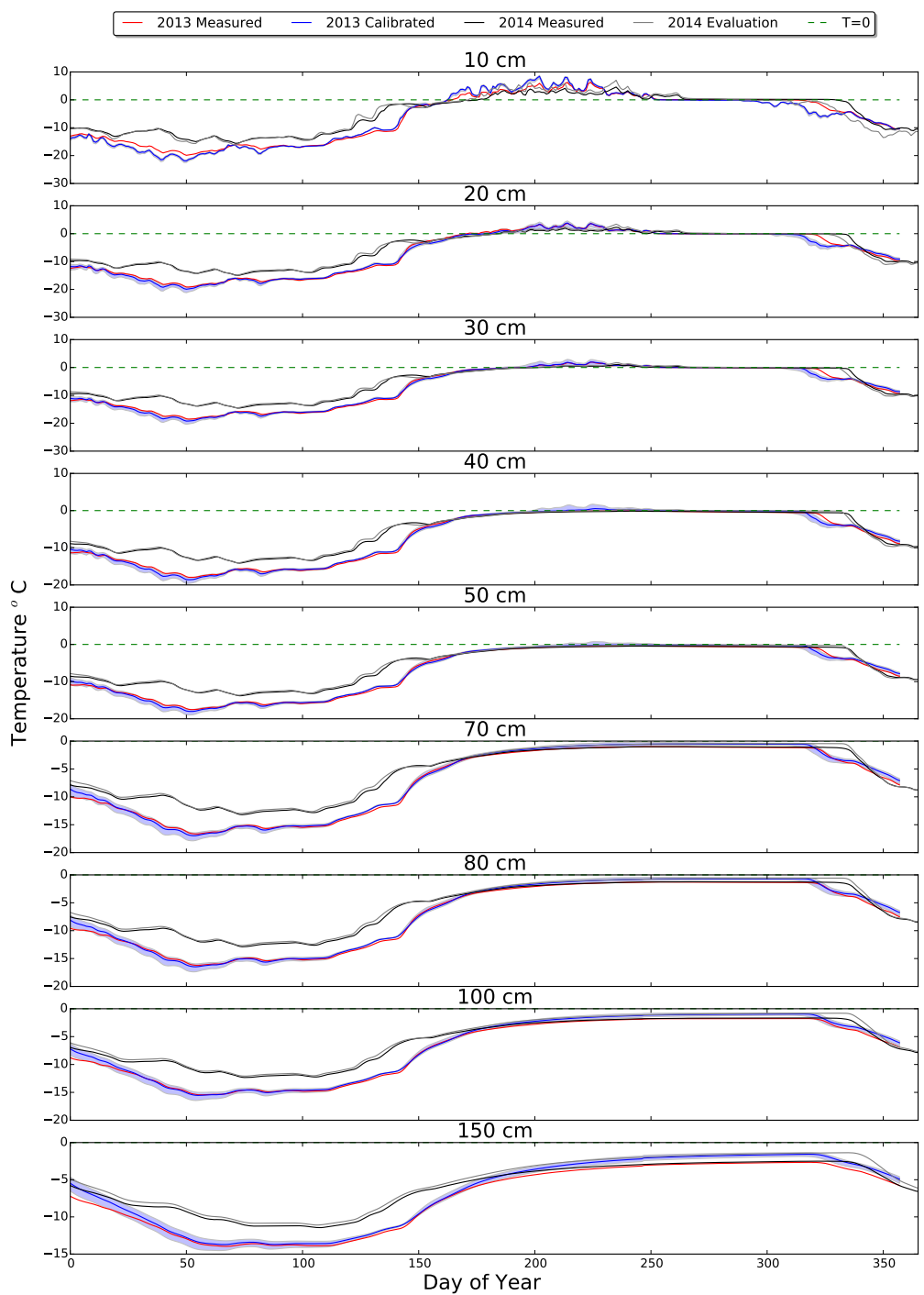

Figure S5: Time-series of temperature at specific depths for the polygonal trough. Calendar year 2013 measured values from the BEO used as calibration targets are shown as a red line, the mean of the NSMC sample as a blue line, and the $95 \%$ confidence band is the shaded light blue region. Calendar year 2014 measured values from the BEO used as evaluation data for the 2013 calibration are shown as a black line. The gray line represents the 2014 simulated temperatures. 

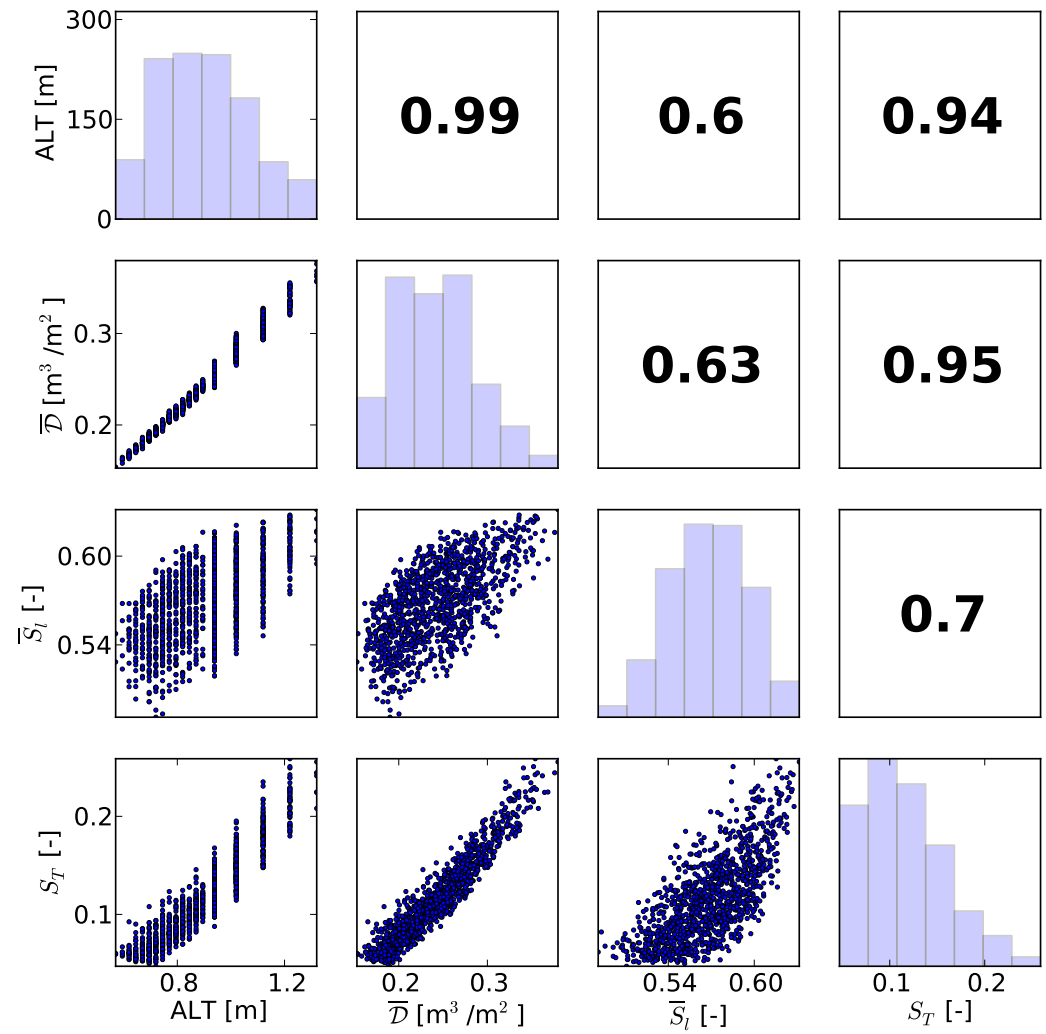

Figure S6: Matrix of paired plots of calibration-constrained ensemble projections for year 2100. Parameter histograms are plotted along the diagonal, paired scatterplots in the lower triangle, and Pearson correlation coefficients in the upper triangle. The range of counts for all histograms are as indicated along the ordinate axis of the upper left plot. 